\title{
Survival in pulmonary hypertension in Spain: insights from the Spanish registry
}

\author{
Pilar Escribano-Subias*, Isabel Blanco\#, Manuel López-Meseguer", \\ Carmen Jimenez Lopez-Guarch*, Antonio Roman ${ }^{\star}$, Pilar Morales ${ }^{+}$, \\ María Jesús Castillo-Palma ${ }^{\S}$, Javier Segovia ${ }^{f}$, Miguel A. Gómez-Sanchez* $^{*}$ \\ and Joan Albert Barberà ${ }^{\#}$ on behalf of the REHAP investigators**
}

ABSTRACT: A pulmonary hypertension (PH) registry (Spanish Registry of Pulmonary Arterial Hypertension) was undertaken to analyse prevalence, incidence and survival of pulmonary arterial hypertension (PAH) and chronic thromboembolic pulmonary hypertension (CTEPH) in Spain, and to assess the applicability of recent survival prediction equations.

Voluntary reporting of previously diagnosed and incident PAH or CTEPH cases (July 2007-June 2008) was performed. Demographic, functional and haemodynamic variables were evaluated.

866 patients with PAH and 162 with CTEPH were included. PAH associated with toxic oil syndrome and pulmonary veno-occlusive disease were reported for the first time in a PAH registry. Estimated prevalences were as follows: $\mathrm{PAH}, 16$ and $\mathrm{CTEPH}, 3.2$ cases per million adult inhabitants (MAI). Estimated incidences were as follows: $\mathrm{PAH}, 3.7$ and $\mathrm{CTEPH}, 0.9$ cases per MAI per yr. 1-, 3- and 5-yr survival was $87 \%, 75 \%$ and $65 \%$, respectively, with no differences between PAH and CTEPH. Male sex, right atrial pressure and cardiac index were independent predictors of death. Matching between observed survival and that predicted by different equations was closer when the characteristics of the cohorts were similar.

Epidemiology and survival of PAH patients in the Spanish registry are similar to recent registries. Characteristics of the population from which survival prediction equations are derived influence their applicability to a different cohort. CTEPH is much less prevalent than PAH, although has a similar survival rate.

KEYWORDS: Chronic thromboembolic pulmonary hypertension, epidemiology, haemodynamics, pulmonary arterial hypertension, risk factors, survival

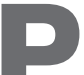
ulmonary hypertension $(\mathrm{PH})$ is a pathophysiological condition defined by the increase of mean pulmonary artery pressure $(P \mathrm{pa})$ that may develop in prevalent heart and lung diseases, or may be present in rare clinical conditions such as pulmonary arterial hypertension (PAH) and chronic thromboembolic pulmonary hypertension (CTEPH) [1].

Rare or orphan diseases benefit from combined national and international efforts to broaden knowledge and develop new therapies. The National Institutes of Health (NIH) registry for PAH [2] was the first comprehensive evaluation of the epidemiology of the idiopathic, familial and anorexigenassociated forms of $\mathrm{PAH}$ in an era lacking in specific $\mathrm{PH}$ therapies. The $\mathrm{NIH}$ registry also facilitated the survival analysis of its cohort and the identification of prognostic variables, making it possible to develop the first predictive survival equation for this condition [3].

Recently, large national observational registries in France [4], Scotland (UK) [5] and the USA [6] have provided information on current $\mathrm{PAH}$ epidemiology, increasing awareness about the disease. Furthermore, these national registries have made possible the reassessment of patient survival under present conditions, leading to the formulation of new predictive survival equations $[7,8]$. Epidemiological data from different countries, sometimes with different healthcare organisation, drug availability and financial outreach, may provide a more comprehensive view of current management of the disease worldwide. Furthermore, there is a need to test the recently developed predictive survival equations [7-9] in populations other than those in which they were
AFFILIATIONS

${ }^{*}$ Cardiology Dept, Hospital Universitario 12 de OctubreREDINSCOR and,

${ }^{f}$ Cardiology Dept, Hospital Universitario Puerta de Hierro, Madrid,

\#Pneumology Dept, Hospital Clínic-CIBERES

-Pneumology Dept, Hospital Universitario Vall d'Hebrón-CIBERES, Barcelona,

+Pneumology Dept, Hospital Universitario La Fe, Valencia, and §Internal Medicine Dept, Hospital Universitario Virgen del Rocío, Seville, Spain.

${ }^{* *} A$ list of all REHAP investigators is given in the Acknowledgements section.

CORRESPONDENCE

P. Escribano-Subias

Pulmonary Hypertension Unit,

Cardiology Dept

Hospital Universitario 12 de Octubre

Complutense University

Madrid

Crtra Andalucia km 5

400

28041 Madrid

Spain

E-mail: pilar.escribano@

telefonica.net

Received:

June 152011

Accepted after revision:

Dec 272011

First published online:

Feb 232012 
formulated in order to validate their general applicability and, hence, their clinical usefulness.

Moreover, epidemiological information on CTEPH is scarce [10]. A multinational registry on CTEPH reported recently provides information on clinical characteristics of the disease, but not on its epidemiology [11].

In order to analyse the prevalence, incidence, survival and clinical management of both PAH and CTEPH in Spain, the Spanish Registry of Pulmonary Arterial Hypertension (REHAP) was established in 2007. In the present study we report the estimated prevalence and incidence, as well as the survival, of adult patients diagnosed with PAH and CTEPH. In addition, we have used this cohort to test whether or not the recently formulated equations are accurate predictors of survival in our population.

\section{METHODS}

The REHAP registry is a voluntary, nonpaid registry that was launched in January 2007. All patients evaluated in 31 hospitals in Spain between January 1, 1998 and June 30, 2008 were included in the registry. Centres reporting patients to the registry covered 15 of the 17 administrative regions of Spain.

Patients diagnosed in the period 1998-2006 were entered retrospectively, and prospectively thereafter. Data were collected by means of electronic data capture (eDC), starting at the initial baseline assessment reported in the medical records. Once the enrolment criteria were satisfied, no tests or study visits were required. Potential data inconsistencies were primarily identified by the eDC system at the point of entry. The protocol was reviewed and approved by the institutional review board of Hospital de Cruces, Bilbao, Spain.

Patients with newly or previously diagnosed $\mathrm{PH}$ were eligible for enrolment if they met the modified definition of $\mathrm{PAH}$ and CTEPH [12] and pre-specified haemodynamic criteria by right heart catheterisation (RHC). Diagnosis of $\mathrm{PH}$ type and specific $\mathrm{PAH}$ subtype was assigned by the investigator reporting the case to the registry. Patients were ineligible if they were aged $<14$ yrs. Patients were classified as newly diagnosed $\mathrm{PH}$ (incident) if the diagnostic RHC took place between July 1, 2007 and June 30, 2008.

To be eligible, RHC must have been performed before the study entry (mean $P$ pa $\geqslant 25 \mathrm{mmHg}$ at rest, pulmonary capillary wedge pressure or left ventricular end-diastolic pressure $\leqslant 15 \mathrm{mmHg}$ and pulmonary vascular resistance (PVR) $\geqslant 3$ Wood units). However, patients with Eisenmenger's syndrome could be diagnosed only by echocardiography. Exclusion criteria were: evidence of left heart disease or respiratory disease (forced vital capacity or forced expiratory volume in $1 \mathrm{~s}<60 \%$ predicted).

Acute vasodilator challenge was performed during RHC with inhaled nitric oxide, adenosine or intravenous prostacyclin, following local standard procedures. The definition of acute vasodilator response has changed over time. Accordingly, in previously diagnosed patients, the definition established by the attending physician was considered, whereas in incident cases, the Venice criteria [13] were adopted.

Survival prediction equations from the NIH [3], the Pulmonary Hypertension Connection (PHC) [14], the French Registry [8] and the Registry to Evaluate Early and Long-Term Pulmonary Arterial Hypertension Disease Management (REVEAL) [7] were used to estimate survival in the idiopathic PAH (IPAH) and hereditary PAH subgroups.

\section{Statistical analysis}

Continuous variables were expressed as mean \pm SD and compared using paired t-tests. One-way ANOVA and Bonferroni's multiple comparison test were used to identify differences among patient groups. Categorical variables were expressed as $n$ (\%) and compared using the Chi-squared test or Fisher's exact test, as appropriate.

Incidence and prevalence were calculated as the ratio of newly diagnosed patients (July 1, 2007-June 30, 2008) or the total number of patients, respectively, and the total Spanish adult population ( $\geqslant 14$ yrs of age), according to the 2008 official population registered in Spain.

Survival analysis was performed using the Kaplan-Meier survival analysis, with the date of entry into the study defined as the date of the first diagnostic RHC or the first visit to the $\mathrm{PH}$ clinic for those patients that did not undergo any initial RHC. All-cause mortality was defined as the end-point and the log-rank test was used for comparison between groups. For survival analysis, the cohort was divided into patients diagnosed before and after July 2007, considering the latter group the incident cases.

Univariate Cox's proportional hazards analysis was used to assess the relationship between PAH and outcome. Co-linearity between variables was examined. Significant variables on the univariate analysis $(\mathrm{p}<0.1)$, along with those variables previously reported to be related with mortality, were included in a forward stepwise multivariate Cox's proportional hazards model in order to identify independent predictors of mortality in the overall $\mathrm{PAH}$ population and specifically in the IPAH group.

The NIH, PHC, French registry and REVEAL equations were applied to estimate survival in the REHAP PAH group in order to assess whether or not these recently formulated equations were accurate predictors of survival in our population.

All p-values were two-sided and a p-value $<0.05$ was considered to be statistically significant. All statistics were performed using SPSS version 17 (SPSS Inc., Chicago, IL, USA).

\section{RESULTS}

\section{Study population}

Initially 1,162 patients were enrolled. However, 134 patients were removed because they did not meet all the inclusion criteria, yielding a final total of 1,028 patients. It should be noted that $61 \%$ of the patients included in the registry were reported by three centres. Clinical and haemodynamic data at the time of diagnosis are shown in table 1.

In incident cases, the delay between the onset of symptoms and diagnosis was 2.2 yrs, whereas in previously diagnosed cases it was 3.7 yrs. Most of the patients had severe symptoms at the time of diagnosis, with $69 \%$ of them in World Health Organization (WHO) functional class (FC) III or IV in the PAH group and $77 \%$ in the CTEPH group. Only $30 \%$ of patients with CTEPH underwent pulmonary endarterectomy (PEA). 


\begin{tabular}{|c|c|c|c|}
\hline \multirow[t]{2}{*}{ TABLE 1} & \multicolumn{3}{|c|}{$\begin{array}{l}\text { Clinical and haemodynamic data at the time of } \\
\text { diagnosis of pulmonary hypertension in } \\
\text { pulmonary arterial hypertension (PAH) and } \\
\text { chronic thromboembolic pulmonary } \\
\text { hypertension (CTEPH) }\end{array}$} \\
\hline & & PAH & СТЕРH \\
\hline \multicolumn{2}{|l|}{ Subjects $n$} & 866 & 162 \\
\hline \multicolumn{2}{|l|}{ Age yrs } & $45 \pm 17$ & $61 \pm 15^{\star}$ \\
\hline \multicolumn{2}{|c|}{ Males \% } & 29 & $40^{\star}$ \\
\hline \multicolumn{2}{|c|}{ Time from symptoms to diagnosis yrs } & $3.5 \pm 6.1$ & $2.7 \pm 4.3^{\star}$ \\
\hline \multicolumn{2}{|l|}{$|-| \mid$} & 31 & 23 \\
\hline \multicolumn{2}{|l|}{ III } & 58 & 68 \\
\hline \multicolumn{2}{|c|}{ IV } & 11 & 9 \\
\hline \multicolumn{2}{|c|}{ Mean Ppa mmHg } & $54 \pm 16$ & $47 \pm 13^{*}$ \\
\hline \multicolumn{2}{|c|}{ Cardiac index L. $\min ^{-1} \cdot \mathrm{m}^{-2}$} & $2.6 \pm 0.9$ & $2.3 \pm 0.5^{\star}$ \\
\hline \multicolumn{2}{|c|}{ PVR Wood units } & $12 \pm 6$ & $10 \pm 5^{\star}$ \\
\hline \multicolumn{2}{|c|}{ Pra mmHg } & $9 \pm 5$ & $8 \pm 6$ \\
\hline \multicolumn{2}{|l|}{ 6MWD m } & $363 \pm 120$ & $317 \pm 112^{*}$ \\
\hline
\end{tabular}

Data are presented as mean \pm SD, unless otherwise stated. WHO: World Health Organization; FC: functional class; Ppa: pulmonary artery pressure; PVR: pulmonary vascular resistance; Pra: right atrial pressure; 6MWD: 6-min walk distance. ${ }^{*}: \mathrm{p}<0.05$ compared with $\mathrm{PAH}$.

The mean 6-min walk distance and haemodynamic data were related to FC in both the PAH and CTEPH groups (table 2).

Acute vasodilator testing was carried out on 643 patients with PAH (75\%) and 91 with CTEPH (52\%). A positive response was observed in $12 \%$ of patients from the PAH incident group, whereas in previously diagnosed $\mathrm{PAH}$ it was reported to be positive in $21 \%$.

\section{Subgroups of PAH}

The distribution and characteristics of patients across the various types of $\mathrm{PAH}$ are shown in table 3 . Among patients with PAH associated with connective tissue diseases (CTDs), systemic sclerosis (61\%) was the most common diagnosis.

In PAH associated with congenital heart disease (CHD) (167 patients), simple type defects were the most frequent forms $(69 \%)$. It is noteworthy that $30 \%$ of patients had PAH after corrective cardiac surgery.
$24(2.3 \%)$ patients displayed two coexisting conditions known to be associated with PAH: HIV infection and portal hypertension were the most common coexisting conditions $(n=8)$.

The clinical and haemodynamic data at diagnosis for each PAH subgroup and CTEPH are displayed in table 3.

\section{Incident versus previously diagnosed cases of $\mathbf{P H}$}

Between July 1, 2007 and June 30, 2008, 167 (16.2\%) patients were newly diagnosed (incident cases), whereas the remaining had been diagnosed before the establishment of the registry.

In previously diagnosed cases, we differentiated between those patients who were alive on July 1, 2007 (prevalent cases) and those who died before that date. The clinical and haemodynamic data at diagnosis for incident and previously diagnosed cases of $\mathrm{PH}$ are summarised in table S1. Incident cases were slightly older with a lower $P$ pa.

\section{Prevalence and incidence}

The overall estimated prevalence of $\mathrm{PH}$ in Spain is 19.2 cases per million adult inhabitants (MAI), based on the 715 cases that were alive between July 1, 2007 and June 30, 2008. The estimated prevalence of PAH was 16 cases per MAI, while the prevalence of CTEPH was 3.2 cases per MAI. Among patients with PAH, the estimated prevalence of IPAH, CHD, CTD and PAH associated with toxic oil syndrome (TOS) were 5.6, 3, 2.5 and 0.5 cases per MAI, respectively.

In this period, the low estimated incidence of $\mathrm{PH}$ was 4.6 cases per MAI per year (PAH 3.7 and CTEPH 0.9 cases per MAI per year). As regards the PAH group, the low estimated incidence of IPAH was 1.2 cases per MAI per year.

\section{Survival}

Over a mean follow-up of $3.7 \pm 4.2$ yrs, 331 (32\%) patients died, while 101 (9.8\%) were lost to follow-up. For survival analysis, the latter were censored at the time of their last visit or observation.

The 1-, 3- and 5-yr survival rates in the different $\mathrm{PH}$ groups are shown in table 4 . Kaplan-Meier survival curves according to different aetiologies are shown in figure 1.

In the subgroup of patients with idiopathic, anorexigen-induced or heritable PAH $(n=314)$, those who showed a positive response $(n=70)$ in the acute vasodilator test were excluded from the survival analysis, to prevent potential bias. Figure $2 \mathrm{a}$

TABLE 2 6-min walk distance (6MWD) and pulmonary haemodynamics in pulmonary arterial hypertension (PAH) and chronic thromboembolic pulmonary hypertension (CTEPH) according to functional class (FC)

\begin{tabular}{|c|c|c|c|c|c|c|c|c|}
\hline & & $\mathrm{PAH}^{\#}$ & & ANOVA p-value & & СТЕРН & & ANOVA p-value \\
\hline WHO FC & $|-| \mid$ & III & IV & & $|-| \mid$ & III & IV & \\
\hline 6MWD m & $442 \pm 97$ & $343 \pm 111$ * & $255 \pm 98^{*}$ & $<0.001$ & $379 \pm 111$ & $309 \pm 10$ * & $210 \pm 82^{*}$ & $<0.001$ \\
\hline PVR Wood units & $9 \pm 5$ & $13 \pm 6^{*}$ & $13 \pm 7^{*}$ & $<0.001$ & $9 \pm 5$ & $10 \pm 5$ & $12 \pm 6$ & 0.083 \\
\hline Pra $\mathrm{mmHg}$ & $8 \pm 5$ & $9 \pm 5$ & $11 \pm 6^{*}$ & 0.004 & $7 \pm 5$ & $8 \pm 5$ & $13 \pm 7^{*}$ & 0.001 \\
\hline
\end{tabular}

Data are presented as mean $\pm \mathrm{SD}$, unless otherwise stated. WHO: World Health Organization; PVR: pulmonary vascular resistance; Pra: right atrial pressure. ${ }^{*}: \mathrm{n}=866$; $\because: \mathrm{n}=162 .{ }^{*}: \mathrm{p}<0.05$ for differences between FC I-II versus FC III and FC IV groups for both aetiologies. 
TABLE 3 Clinical and haemodynamic data at the time of diagnosis in pulmonary arterial hypertension (PAH) subtypes and chronic thromboembolic pulmonary hypertension (CTEPH)

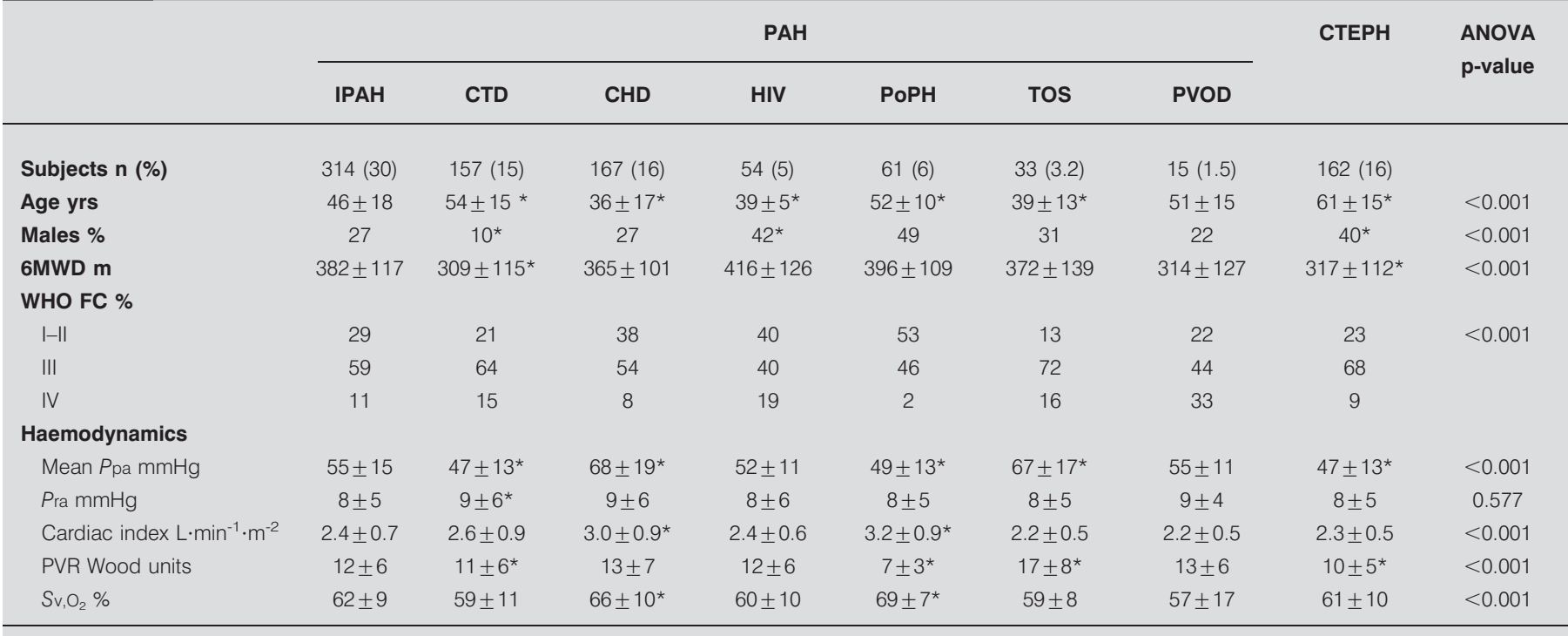

Data are presented as mean \pm SD, unless otherwise stated. IPAH: idiopathic PAH; CTD: connective tissue disease; CHD: congenital heart disease; PoPH: portopulmonary hypertension; TOS: toxic oil syndrome; PVOD: veno-occlusive disease; 6MWD: 6-min walk distance; WHO: World Health Organization; FC: functional class; Ppa: pulmonary artery pressure; Pra: right atrial pressure; PVR: pulmonary vascular resistance; $\mathrm{Sv}_{\mathbf{1}} \mathrm{O}_{2}$ : mixed-venous oxygen saturation. ${ }^{*}$ : $\mathrm{p}<0.05$ compared with IPAH.

shows predicted survival using the NIH and the PHC Registry equations in the IPAH nonresponders population.

In order to test the applicability of the French registry equation to estimate survival in our cohort, we restricted the analysis to patients with IPAH diagnosed up to 48 months before June 30, 2008 (45 incident and 104 diagnosed in the 36 months previous). Figure $2 \mathrm{~b}$ shows both the observed and the estimated survival curves. Survival predicted by the equation derived from the French registry slightly infra-estimated the actual survival of the REHAP cohort the first 2 yrs $(\mathrm{p}=0.019)$.

Furthermore, we also considered the applicability of the REVEAL equation to estimate the 1-yr survival. In order to achieve comparable populations, we excluded from the analysis those patients with TOS $(n=33)$, pulmonary veno-occlusive

\begin{tabular}{llllll}
\hline TABLE $\mathbf{4}$ & $\begin{array}{l}\text { Survival rates in the Spanish Registry of } \\
\text { Pulmonary Arterial Hypertension (REHAP) } \\
\text { registry }\end{array}$ & & & & \\
& Subjects $\mathbf{n}$ & $\mathbf{1} \mathbf{~ y r}$ & $\mathbf{3}$ yrs & $\mathbf{5}$ yrs \\
\hline & 1028 & 87 & 75 & 65 \\
All patients & 861 & 87 & 75 & 65 \\
Diagnosed before July $\mathbf{2 0 0 7}$ & 167 & 88 & 74 & \\
Diagnosed after July $\mathbf{2 0 0 7}$ & 162 & 93 & 75 & 65 \\
CTEPH & 314 & 89 & 77 & 68 \\
IPAH & & & & \\
\hline
\end{tabular}

Data are presented as \%, unless otherwise stated. CTEPH: chronic thromboembolic pulmonary hypertension; IPAH: idiopathic pulmonary arterial hypertension disease (PVOD) $(n=15)$, and also patients with fewer than seven factors contained in the REVEAL formula $(n=108)$, which is the minimum necessary to maintain the reliability of the results. The 1-yr survival in the 713 remaining patients with PAH estimated by the REVEAL equation was $92 \%$, whereas the observed

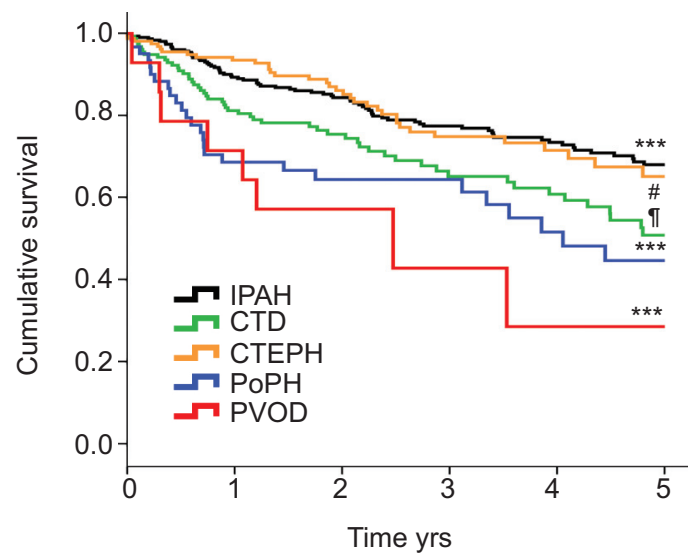

$\begin{array}{lccccccc}\text { At risk n } & & & & & & & \text { p-value\# } \\ \text { IPAH } & 314 & 304 & 236 & 179 & 136 & 108 & \\ \text { CTD } & 157 & 149 & 98 & 67 & 46 & 37 & 0.023 \\ \text { CTEPH } & 162 & 153 & 117 & 82 & 51 & 34 & 0.951 \\ \text { PoPH } & 61 & 58 & 34 & 26 & 21 & 14 & <0.001 \\ \text { PVOD } & 15 & 14 & 8 & 4 & 3 & 2 & <0.001\end{array}$

FIGURE 1. Kaplan-Meier estimates of 5-yr survival from time of diagnosis in different pulmonary hypertension subtypes. IPAH: idiopathic pulmonary arterial hypertension; CTD: connective tissue disease; CTEPH: chronic thromboembolic pulmonary hypertension; PoPH: portopulmonary hypertension; PVOD: pulmonary veno-occlusive disease. The $\mathrm{p}$-value for the overall comparison is $<0.001$. \#. compared with IPAH. 

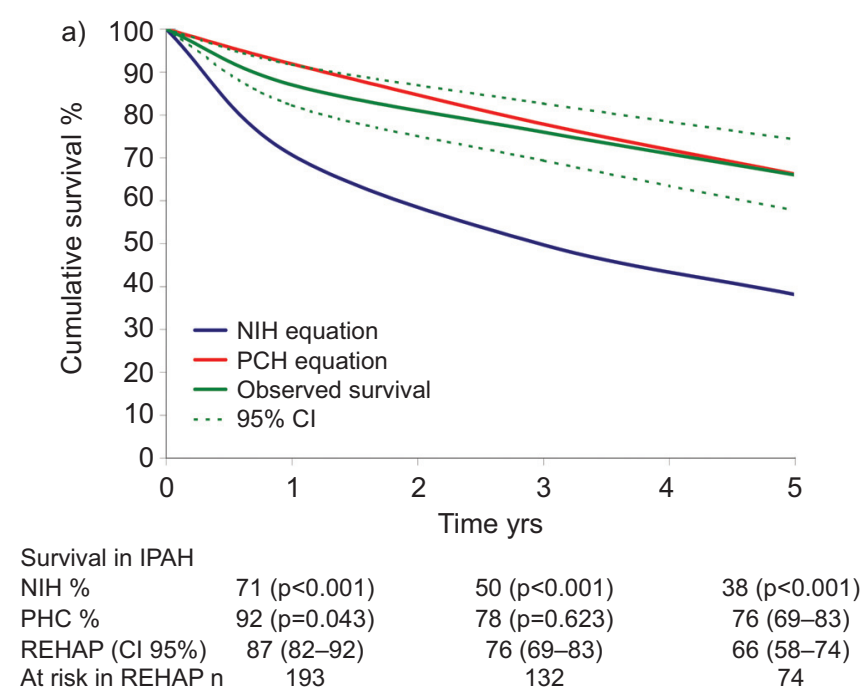

At risk in REHAP n $193 \quad 132$

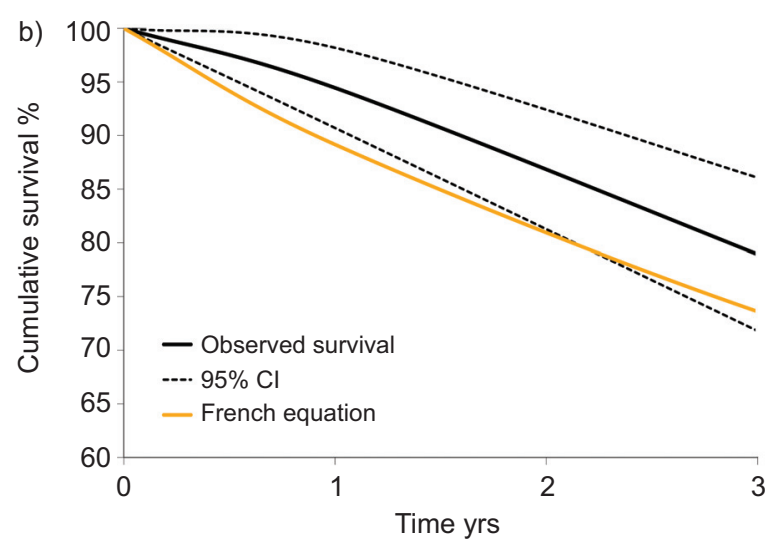

Survival in IPAH

French registry \%

REHAP (CI 95\%)

At risk in REHAP n $73(p=0.174)$

$78(72-86)$ 114
FIGURE 2. a) Observed and estimated survival (National Institutes of Health (NIH) and Pulmonary Hypertension Connection (PHC) equations) of idiopathic pulmonary arterial hypertension (IPAH) nonresponders in the Spanish Registry of Pulmonary Arterial Hypertension (REHAP) registry. b) Observed and estimated survival (French equations) of IPAH cases diagnosed $<48$ months before June 30 , 2008 in the REHAP registry.

survival (number at risk 686) was significantly lower $(88 \%, 95 \%$ CI 85-91\%; $p=0.001$ ). However, when the REVEAL risk equation was applied exclusively in the incident group predicted survival was 93\%, whereas observed 1-yr survival (number at risk $=118$ ) was only slightly lower, without significant difference (88\%, 95\% CI 82-94\%; $\mathrm{p}=0.157$ ).

In the univariate analysis, age, male sex, WHO FC III-IV, pericardial effusion, reduced exercise capacity, increased right atrial pressure (Pra), PVR, heart rate, reduced \% predicted carbon monoxide diffusing capacity, cardiac index and cardiac output were all associated with an increased risk of death. However, time since diagnosis and blood pressure were not associated with an increased risk. Among subtypes of $\mathrm{PAH}$, PAH associated with CTD, portal hypertension and PVOD were all associated with an increased risk of death. In contrast, $\mathrm{PAH}$ associated with CHD was associated with a lesser risk of death when compared with IPAH (table S2).

In the multivariate analysis (table 5), only male sex, WHO FC, $P$ ra and cardiac index remained independent predictors of death in $\mathrm{PAH}$. With regard to PAH subtypes, $\mathrm{PAH}$ associated with CTD, portal hypertension or PVOD also remained as independent predictors of death.

\section{DISCUSSION}

The REHAP registry is one of the largest registries of patients with PAH reported to date and also provides novel epidemiological information on CTEPH. It is the first registry including PVOD and offers information on PAH associated with the epidemic caused by the ingestion of denatured rapeseed oil that took place in Spain in 1981. It has also provided the opportunity to examine the performance of recently reported survival prediction equations in an independent population.

The design of the registry, which included referral centres and community hospitals in Spain over a prolonged period of time (1998-2008), closely reflects the general practice over these 10 yrs.

\section{Epidemiology of $\mathbf{P H}$ in Spain}

The observed incidence and prevalence of IPAH in REHAP are similar to those observed in the French registry [4] and clearly lower than those described in the Scottish registry [5]. Methodological differences in the criteria used to include cases may account for these differences. Presumably, both the French and the current registry may underestimate these figures in their respective countries.

The incidence and prevalence of PAH associated with other conditions varies enormously between registries, mainly due to registry design and PAH subtypes that are included, particularly with respect to the number of cases of $\mathrm{PAH}$ associated with $\mathrm{CHD}$. In the majority of registries published to date, the number of patients with CHD is relatively low, probably reflecting a lack of interaction between physicians caring for patients with CHD and $\mathrm{PH}$ units. In the recently published Belgian registry on CHD [15], the estimated prevalence of Eisenmenger's syndrome was 11 cases per MAI, clearly higher than the prevalence observed in the REHAP (2.9 cases per MAI), even after taking into account the fact that patients with PAH after corrective surgery were not included in the Belgian registry.

\section{Pulmonary veno-occlusive disease}

The true incidence and prevalence of PVOD is unknown and probably underestimated, because many cases might be misclassified as IPAH. There is no information about the epidemiology of PVOD in any PH registry. Prevalence in the current registry was 0.16 cases per MAI, representing $1.5 \%$ of all $\mathrm{PH}$ subtypes. Patients with PVOD had more severe hypoxaemia, lower carbon monoxide diffusion capacity, absence of vasodilator response and the worst survival compared with patients with IPAH, which is in line with previous reports [16].

\section{PH associated with TOS}

$\mathrm{PH}$ associated with TOS is a particular subtype of PAH related to an epidemic produced by the ingestion of rapeseed oil that had been denatured for industrial use, which took place in Spain in May, 1981 and affected $>20,000$ people. $\mathrm{PH}$ developed 
TABLE 5 Multivariate predictors of death in Cox's proportional hazards analysis

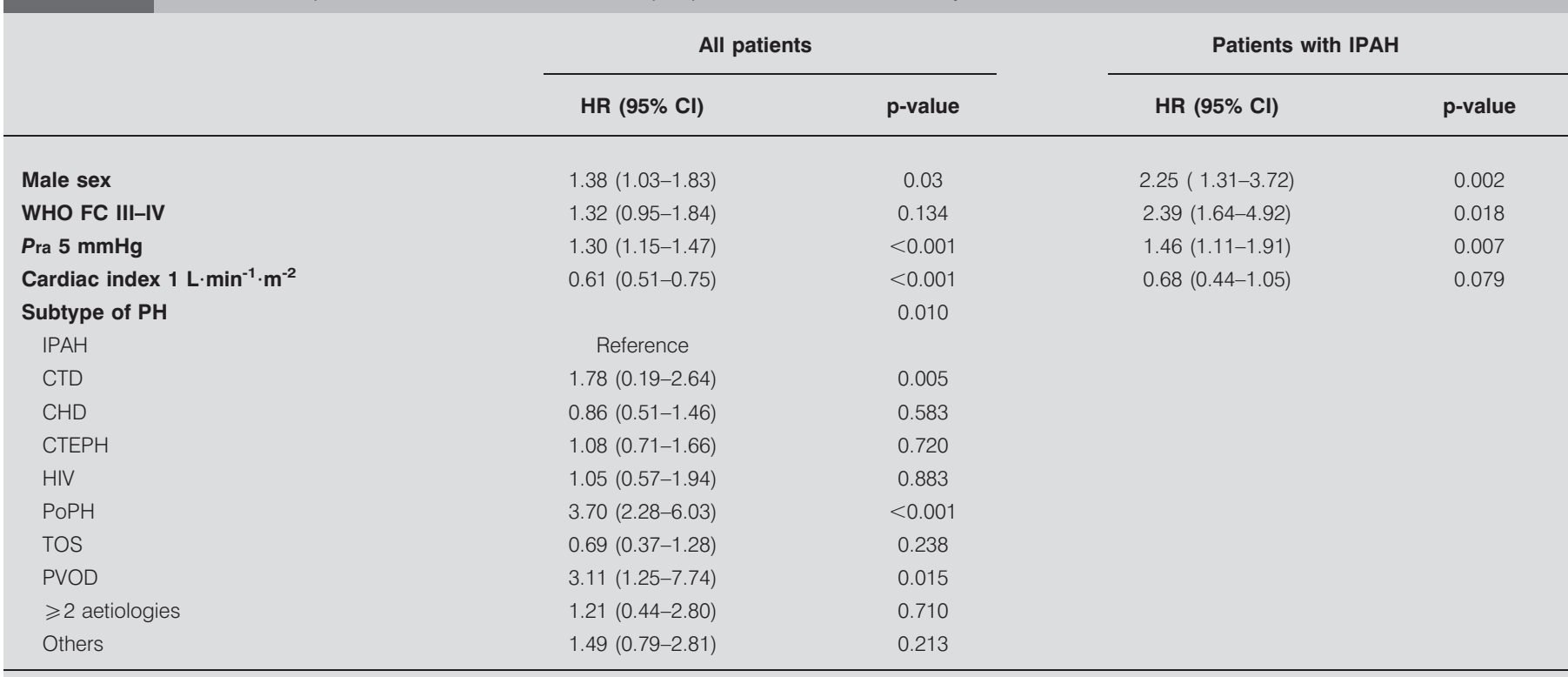

IPAH: idiopathic pulmonary arterial hypertension; HR: hazard ratio; WHO: World Health Organization; FC: functional class; Pra: right atrial pressure; PH: pulmonary hypertension; CTD: connective tissue disease; CHD: congenital heart disease; CTEPH: chronic thromboembolic pulmonary hypertension; PoPH: portopulmonary hypertension; TOS: toxic oil syndrome; PVOD: pulmonary veno-occlusive disease

in $\sim 20 \%$ of the patients, although in most of them it regressed spontaneously. Nevertheless, in a small number of patients $(0.2 \%)$ it progressed to a malignant course, leading rapidly to death in the following years. A small group of patients $(n=33)$ not initially diagnosed with severe PAH developed the disease after a latency period of 15-20 yrs. They all share common characteristics: all of them belong to the registered TOS population group (official Spanish census), approximately half of them had mild PAH in the acute phase of the disease and, in general, they had a good quality of life until the diagnosis of severe PAH (some females had successful full-term pregnancies during this period). The last diagnosis of a patient with TOS-associated PAH was in 2007 (26 yrs after the epidemic). In contrast with other registries [4, 6], PAH induced by anorexigen use appears to be extremely rare in Spain.

\section{CTEPH}

Information about the incidence and prevalence of CTEPH is sparse and we have only an indirect estimate carried out by CONDLIFFE et al. [10], where the estimated incidence was 1.75 cases per MAI. In the REHAP registry, the incidence was 0.9 cases per MAI per year (clearly lower than the UK results), and the prevalence was 3.2 cases per MAI, which is the only reported figure to date.

\section{Factors affecting survival}

All recent registries $[4-6,14]$ and the current registry show that $\mathrm{PAH}$ is still detected late in the course of the disease.

In our registry, among patients with $\mathrm{PAH}$, male sex, lower cardiac index and higher Pra were independent predictors of death. Regarding the aetiology of $\mathrm{PH}$, three subtypes remain as independent predictors of outcome: portopulmonary hypertension,
PVOD and CTD. The importance of the haemodynamic values has been previously published $[17,18]$. However, the more negative prognosis associated with male sex, a finding that has also been reported in the REVEAL [7] and French registries [8], does not have any clear explanation.

In our cohort, portopulmonary hypertension was found to be an independent predictor of death, as it has also been reported in the REVEAL registry [7]. In contrast, no difference in mortality between IPAH and porto-pulmonary hypertension was observed in the French registry [19]. One potential explanation for such difference might be related to the severity of underlying hepatic disease, which unfortunately is not reported in $\mathrm{PAH}$ registries.

CTD was also found to be an independent risk factor for mortality, in agreement with all previous registries $[7,14,19,20]$.

\section{Survival prediction}

In our cohort of patients with IPAH, observed survival was $89 \%$ and $77 \%$ at 1 and 3 yrs, respectively. These findings are similar to those observed in the recent USA PHC registry [9] and are better than those reported $>20$ yrs ago in the $\mathrm{NIH}$ registry [2]. Likewise, the 3 -yr survival rate of IPAH nonresponders to the vasodilator test in the REHAP registry matches perfectly with the predicted survival using the PHC equation [9]. Although some degree of improvement in survival compared with historical cohorts can be expected due to advances in general patient care and improved living circumstances, the magnitude seen here suggests an effect derived from specific $\mathrm{PH}$ therapy.

The overall survival of patients with PAH in REHAP was similar to that of the PHC, REVEAL and French registries (table S3). 
However, differences in registry design might influence the potential applicability of a given survival prediction equation to populations different from those where the equation was derived. The REHAP registry included 1,028 consecutive patients diagnosed in Spain over one decade (1998-2008). As mentioned, observed survival in this cohort fits quite well with that predicted from the PHC registry equation [9], which also covers a broad time span (1991-2007). In contrast, survival in the REHAP registry, restricted to patients with IPAH diagnosed $<48$ months before June 30, 2008, was slightly better in the first 2 yrs than that predicted using the French registry equation [8]. Presumably, this could be explained because patients in the REHAP were diagnosed between 2004 and 2008, and had less severe disease at diagnosis than patients in the French registry, who were diagnosed between 1999 and 2003 [19]. Such differences in the time of diagnosis could be important in $\mathrm{PAH}$, where the therapeutic approach has been refined in the past 5 yrs, as $\mathrm{PH}$ units have gained more experience. Currently, treatment is started at a less severe disease stage and therapeutic goals are more ambitious [21]. All these factors could explain the observed differences in survival.

Regarding the equation derived from the REVEAL registry [7], the 1-yr survival observed in the REHAP registry for all PAH cases was significantly lower than that predicted by this equation. This fact may be related to differences in population selection: whereas the REVEAL registry included $85 \%$ of prevalent cases, in the REHAP registry sequentially diagnosed patients were enrolled, including both alive (prevalent) and deceased patients, over a long period of time. Moreover, the REHAP registry extends back to 1998 , whereas the REVEAL registry only extends back to 2003. This is important because prior to 2003 the only treatments that were available were epoprostenil and treprostinil. Presumably, these differences could explain the observed differences when the whole REHAP cohort is considered, whereas 1-yr survival of incident cases in the REHAP registry fits quite well with that predicted by the REVEAL equation. All in all, these observations suggest a beneficial impact on PAH survival of contemporary therapeutic strategy.

\section{Survival in CTEPH}

Until recently it was thought that CTEPH had a better prognosis than IPAH, partly due to the fact that it is the only potentially curable form of $\mathrm{PH}$. The overlap in the survival curves of CTEPH and IPAH observed in our study was unanticipated. Survival of the CTEPH cohort, of which only 30\% underwent PEA, was similar to that reported in the Swiss registry [22] (10\% PEA) and only slightly lower than that experienced in the UK (50\% PEA) [10]. However, the 5-yr survival after PEA of patients who survived up to 3 months after surgery was $90 \%$, with a remarkable improvement in functional capacity and haemodynamic profile, which was markedly better than that of nonsurgical patients [23-25]. Thus, continued efforts to consider PEA in CTEPH treatment are needed.

Several limitations should be considered when interpreting data from our study. This is an observational and voluntary registry with a large number of patients entered retrospectively; this can lead to missing or inconclusive data for analysis. However, these limitations could be minimised, as two-thirds of the patients included in REHAP had been prospectively collected in local databases, which were included in the registry database. In addition, although all $\mathrm{PH}$ centres included patients in the registry, we cannot rule out that patients with $\mathrm{PH}$ treated in small hospitals were not reported to the registry, which works on a voluntary basis. However, since all $\mathrm{PH}$ centres were included, the REHAP population is representative of the whole Spanish PAH population. Furthermore, it should be also taken into consideration that these problems are unavoidable when doing a multicentre national registry.

The Spanish PH registry covers a long period of time, almost a decade. The management of the disease has changed considerably over the course of these years. Initially, the time between the onset of symptoms and diagnosis was $>3$ yrs. Accordingly, some of the most aggressive cases may have died before being diagnosed, and therefore before being included in the registry. This may have led to an underestimate of mortality.

In summary, the present study shows striking similarities in PAH epidemiology, as well as in evolution and survival, between patients in Spain and those reported in recent European and American registries. Results of the REHAP registry confirm significant survival improvement with current treatment strategies and provide evidence on the applicability of recently reported survival prediction equations to different populations, although differences intrinsic to registry design should be taken into account. Overall, our results support the validity of multicentre international clinical trials and indicate that, despite differences in health systems and populations, the evolution of the disease is quite homogeneous when access to advanced specific therapies is guaranteed.

\section{SUPPORT STATEMENT}

The REHAP registry is sponsored by an unrestricted educational grant from Bayer Schering Pharma.

\section{STATEMENT OF INTEREST}

Statements of interest for J. Segovia and M.A. Gómez-Sanchez, and for the study itself can be found at www.erj.ersjournals.com/site/misc/ statements.xhtml

\section{ACKNOWLEDGEMENTS}

We thank the Registry Coordinating Centre, S\&H Medical Science Service (Madrid, Spain), for their quality control, logistic and administrative support, and S. Ortiz (Universidad Autónoma de Madrid, Madrid, Spain) and Statistical Advisor for S\&H Medical Science Service, for the statistical analysis.

Members of the Registro Español de Hipertensión Arterial Pulmonar (REHAP) Group are as follows: S. Alcolea, Hospital Universitario La Paz, Madrid; L. Alonso-Pulpón, Hospital Universitario Puerta de Hierro, Madrid; C. Álvarez, Hospital Universitario Central de Asturias, Oviedo; J.A. Barberà and I Blanco, Hospital Clínic, Barcelona; E. Bollo, Hospital de León, León; M.J. Castillo-Palma, Hospital Universitario Virgen del Rocío, Seville; D. Crémer, Hospital Son Llátzer, Palma de Mallorca, Spain; J.L. de la Cruz, Hospital Regional Universitario Carlos Haya, Málaga; R. del Pozo, Hospital Clínic, Barcelona; E. Díaz, Hospital Universitario de Guadalajara, Guadalajara; C. Disdier, Hospital de San Pedro de Alcántara, Cáceres; J.A. Domingo, Hospital Universitario Miguel Servet, Zaragoza; L. Dos, Hospital Vall dHebrón, Barcelona; V. Egurbide, Hospital de Cruces, Bilbao; P. Escribano-Subias, Hospital Universitario 12 de Octubre, Madrid; J. Gaudo, Hospital Universitario Ramón y Cajal, Madrid; F.J. García, Hospital Universitario Virgen del Rocío, Seville; S. García, Hospital de León, León; M. Gómez, Hospital Universitario Puerta de Hierro, Madrid; F.J. Guerra, Hospital 
Universitario Insular de Gran Canaria, Las Palmas; F. Hernández, Hospital General Universitario de Valencia, Valencia; A. Lara, Hospital Universitario de Canarias, Tenerife; M. Lázaro, Hospital Virgen de la Salud, Toledo; J.L. Lobo, Hospital de Txagorritxu, Vitoria; P. Morales, Hospital Universitario La Fe, Valencia; M.A. Nieto, Hospital Clínico San Carlos, Madrid; J.M. Oliver, Hospital Universitario La Paz, Madrid; I. Otero, Hospital Juan Canalejo, A Coruña; M. Ramón, Hospital General Universitario de Valencia, Valencia; V. Roig, Hospital Clínico Universitario de Valladolid, Valladolid; A. Román, Hospital Vall dHebrón, Barcelona; M.J. Ruiz-Cano, Hospital Universitario 12 de Octubre, Madrid; E. Sala, Hospital Universitario Son Espases, Palma; G.J. Samper, Hospital General Universitario de Valencia, Valencia; J. Sánchez, Hospital Universitario Virgen del Rocío, Seville; J. Segovia, Hospital Universitario Puerta de Hierro, Madrid; M. Subirana, Hospital de Sant Pau, Barcelona; and L. Tomás, Hospital de Txagorritxu, Vitoria, all Spain.

\section{REFERENCES}

1 Galiè N, Hoeper M, Humbert M, et al. Guidelines for the diagnosis and treatment of pulmonary hypertension. Eur Heart J 2009; 30: 2493-2537.

2 Rich S, Dantzker DR, Ayres SM, et al. Primary pulmonary hypertension. A national prospective study. Ann Intern Med 1987; 107: 216-223.

3 D'Alonzo GE, Barst RJ, Ayres SM, et al. Survival in patients with primary pulmonary hypertension. Results from a national prospective registry. Ann Intern Med 1991; 115: 343-349.

4 Humbert $\mathrm{M}$, Sitbon $\mathrm{O}$, Chaouat $\mathrm{A}$, et al. Pulmonary arterial hypertension in France: results from a national registry. $A m$ Respir Crit Care Med 2006; 173: 1023-1030.

5 Peacock AJ, Murphy NF, McMurray JJV, et al. An epidemiological study of pulmonary arterial hypertension. Eur Respir J 2007; 30: 104-109.

6 Badesch DB, Raskob GE, Elliott CG, et al. Pulmonary arterial hypertension: baseline characteristics from the REVEAL registry. Chest 2010; 137: 376-387.

7 Benza RL, Miller DP, Gomberg-Maitland M, et al. Predicting survival in pulmonary arterial hypertension: insights from the Registry to Evaluate Early and Long-Term Pulmonary Arterial Hypertension Disease Management (REVEAL). Circulation 2010; 122: 164-172.

8 Humbert M, Sitbon O, Yaïci A, et al. Survival in incident and prevalent cohorts of patients with pulmonary arterial hypertension. Eur Respir J 2010; 36: 549-555.

9 Thenappan T, Shah SJ, Rich S, et al. Survival in pulmonary arterial hypertension: a reappraisal of the NIH risk stratification equation. Eur Respir J 2010; 35: 1079-1087.

10 Condliffe R, Kiely DG, Gibbs JS, et al. Improved outcomes in medically and surgically treated chronic thromboembolic pulmonary hypertension. Am J Respir Crit Care Med 2008; 177: 1122-1127.
11 Delcroix M, Lang I, Mayer E, et al. Chronic thromboembolic pulmonary hypertension: results from an international prospective registry. Circulation 2011; 124: 1973-1981.

12 Badesch BD, Champion HC, Gómez-Sánchez MA, et al. Diagnosis and assessment of pulmonary arterial hypertension. J Am Coll Cardiol 2009; 54: Suppl. 1, S55-S56.

13 Galiè N, Hoeper M, Humbert M, et al. Guidelines for the diagnosis and treatment of pulmonary hypertension. Eur Heart J 2009; 30: 2493-2537.

14 Thenappan $\mathrm{T}$, Shah SJ, Rich S, et al. A USA-based registry for pulmonary arterial hypertension: 1982-2006. Eur Respir J 2007; 30: 1103-1110.

15 Van de Bruaene A, Delcroix M, Pasquet A, et al. The Belgian Eisenmenger syndrome registry: implications for treatment strategies? Acta Cardiol 2009; 64: 447-453.

16 Montani D, Price LC, Dorfmuller P, et al. Pulmonary venoocclusive disease. Eur Respir J 2009; 33: 189-200.

17 McLaughlin VV, Shillington A, Rich S. Survival in primary pulmonary hypertension: the impact of epoprostenol therapy. Circulation 2002; 106: 1477-1482.

18 Sitbon $\mathrm{O}$, Humbert $\mathrm{M}$, Nunes $\mathrm{H}$, et al. Long-term intravenous epoprostenol infusion in primary pulmonary hypertension: prognostic factors and survival. I Am Coll Cardio 2002; 40: 780-788.

19 Humbert M, Sitbon O, Chaouat A, et al. Survival in patients with idiopathic, familial, and anorexigen-associated pulmonary arterial hypertension in the modern management era. Circulation 2010; 122: 156-163.

20 McLaughlin VV, Suissa S. Prognosis of pulmonary arterial hypertension: the power of clinical registries of rare diseases. Circulation 2010; 122: 106-108.

21 Hoeper MM, Markevych I, Spiekerkoetter E, et al. Goal-oriented treatment and combination therapy for pulmonary arterial hypertension. Eur Respir J 2005; 26: 858-863.

22 Tueller C, Stricker H, Soccal P, et al. Epidemiology of pulmonary hypertension: new data from the Swiss registry. Swiss Med Weekly 2008; 138: 379-384

23 Mayer E, Jenkins D, Lindner J, et al. Surgical management and outcome of patients with chronic thromboembolic pulmonary hypertension: results from an international prospective registry. J Thorac Cardiovasc Surg 2011; 141: 702-710.

24 Jaff MR, McMurtry MS, Archer SL, et al. Management of massive and submassive pulmonary embolism, iliofemoral deep vein thrombosis, and chronic thromboembolic pulmonary hypertension: a scientific statement from the American Heart Association. Circulation 2011; 123: 1788-1830.

25 Couturaud F, Frachon I, Leroyer C. Chronic thromboembolic pulmonary hypertension: atribute to pulmonary endarterectomy. Eur Respir J 2009; 33: 230-232. 